\title{
Partial Breast Irradiation
}

National Cancer Institute

\section{Source}

National Cancer Institute. Partial Breast Irradiation. NCI Thesaurus. Code C116439.

External beam radiation that is focused on a specific part of the breast. 